\title{
Expansion and Hepatocytic Differentiation of Liver Progenitor Cells In Vivo Using a Vascularized Tissue Engineering Chamber in Mice
}

\author{
Natasha Forster, M.D., Jason A. Palmer, B.Sc. (Hons), ${ }^{1}$ George Yeoh, Ph.D., ${ }^{2}$ \\ Wei-Chen Ong, M.D., Geraldine M. Mitchell, Ph.D., John Slavin, MBBS, FRCPA, ${ }^{3}$ \\ Janina Tirnitz-Parker, Ph.D., ${ }^{2}$ and Wayne A. Morrison, M.D., B.S. ${ }^{1}$
}

Current cell-based treatment alternatives to organ transplantation for liver failure remain unsatisfactory. Hepatocytes have a strong tendency to dedifferentiate and apoptose when isolated and maintained in culture. In contrast, liver progenitor cells (LPCs) are robust, easy to culture and have been shown to replace damaged hepatocytes in liver disease. In this study we investigate whether isolated LPCs can survive and differentiate toward mature hepatocytes in vivo when implanted into a heterotopic mouse tissue engineering chamber model. Healthy Balb/c mice and those put on a choline-deficient ethionin-supplemented diet to induce chronic liver disease were implanted with a tissue engineering chamber based on the epigastric flow through pedicle model, containing either $1 \times 10^{6}$ LPCs suspended in Matrigel, or LPC-spheroids produced by preculture for 1 week in Matrigel. Four weeks after implantation the chamber contents were harvested. In all four groups, progenitor cells persisted in large numbers to 4 weeks and demonstrated evidence of considerable proliferation judged by Ki67-positive cells. Periodic acid Schiff staining demonstrated differentiation of some cells into mature hepatocytes. Constructs grown from LPC-spheroids demonstrated considerably greater LPC survival than those from LPCs that were grown as monolayers and implanted as dissociated cells. The combined use of LPC spheroids and the vascularized chamber model could be the basis for a viable alternative to current treatments for chronic liver failure.

\section{Introduction}

$\mathbf{T}$ HE TREATMENT OF acute or chronic organ failure remains one of the greatest therapeutic challenges in medicine. Efforts to circumvent the necessity for the ever-diminishing number of donor organs have spurred research into regenerative techniques. The liver has an immense innate regenerative capacity, whereby acute injury results in an increased proliferation of the remaining healthy hepatocytes to replace the lost cell mass. Such is the regenerative power, that even a partial hepatectomy of up to $70 \%$ can be compensated merely by proliferation. ${ }^{1}$ The first attempts to take advantage of this property for the treatment of liver failure date back to 1977, when Groth et al. transplanted hepatocytes into the Gunn rat-an animal model for Crigler-Najjar syndromeresulting in decreased hyperbillirubinemia. ${ }^{2}$ Despite these and other initial encouraging findings, the use of mature hepatocytes as an alternative to whole-organ transplantation has been limited by their fragility after isolation. The quality and yield of functional cells varies immensely from preparation to preparation, and in vitro, the life-span of hepatocytes is significantly reduced. Their exceedingly low proliferation rate in culture and propensity to dedifferentiate makes it very difficult to generate sufficiently large numbers of functional cells for cell therapy applications. ${ }^{3}$ Not only are they highly sensitive to freeze-thaw procedures, but also in culture isolated hepatocytes tend to apoptose rapidly. This phenomenon is chiefly due to their complex intercellular attachment dependency. ${ }^{4-6}$ Despite the development of highly innovative culture methods, including three-dimensional matrices and the design of bioartificial liver devices, the success of cell-based treatments for liver failure using mature hepatocytes remains very limited. ${ }^{7}$ As a consequence, research has been driven toward alternate cell sources such as stem cells (mesenchymal and hematopoietic) and liver progenitor cells (LPCs).

\footnotetext{
${ }^{1}$ Bernard O'Brien Institute of Microsurgery, St. Vincent's Hospital, Fitzroy, Australia.

${ }^{2}$ Centre for Medical Research, Western Australian Institute for Medical Research and School of Biomedical, Biomolecular and Chemical Sciences, University of Western Australia, Crawley, Australia.

${ }^{3}$ Department of Pathology, St. Vincent's Hospital, Melbourne, Australia.
} 
In the healthy liver, hepatocytes replicate in a regulated manner maintaining a sufficient cell mass to meet the liver's functional demand. When this process is inhibited, such as in the case of both chronic and carcinogenic injury, a distinct population of cells, the so-called oval or LPCs, proliferate and infiltrate the liver parenchyma from their origin in terminal bile ducts. ${ }^{8}$ Not only can these cells be relatively easily isolated and readily expanded in vitro, but they are also bipotential as they can differentiate into both hepatocytes and cholangiocytes in vivo and in vitro. ${ }^{9}$ Further, after proliferation and differentiation, a pool of progenitor cells is retained. These combined properties have encouraged attempts to use LPCs in the context of liver cell transplantation. Thus far, there have been only few reports of attempts to engineer liver tissue from LPCs in vivo. ${ }^{10,11}$ Based on our previous experience cultivating organoids from other cell types in vivo using a vascularized chamber model, ${ }^{12}$ we undertook this study to determine whether such a tissue engineering construct with a defined vascular supply can support and/or induce the growth and differentiation of LPCs toward functional mature hepatocytes in a mouse model.

\section{Materials and Methods}

\section{Animals and anesthesia}

All experiments were performed with the approval of the St. Vincent's Hospital Animal Ethics Committee, under the National Health and Medical Research Council Australia guidelines. Wild-type male Balb/c mice (Animal Resource Centre) weighing 18-24 g were used in all experiments. They were housed in an approved facility, on a $12 \mathrm{~h}$ day/night cycle, and given food and water ad libitum. All experiments were performed with the mice placed under general anesthesia (chloral hydrate administered intraperitoneally at $0.4 \mathrm{mg} / \mathrm{g}$ body weight).

\section{Choline-deficient, ethionin-supplemented diet}

The choline-deficient ethionin-supplemented (CDE) diet is a well-established model for inducing chronic liver injury through hepatic steatosis, ${ }^{13}$ which induces promitotic cytokines, including tumor necrosis factor, interleukin-6, interferon gamma, oncostatin $\mathrm{M}$, and lymphotoxin beta. ${ }^{14}$ It has also successfully been used to investigate oval cell/liver progenitor biology by facilitating their isolation, and culture and proliferation and differentiation in vivo. ${ }^{15}$

The mice in two of the four experimental groups were subjected to the CDE diet for 4 weeks. They were fed a 1:1 mixture of $50 \%$ choline-deficient chow (MP, Cat. No. 960209) and normal powdered chow. DL-Ethionine (Sigma Aldrich) was administered separately in the drinking water. ${ }^{13}$

\section{Surgical technique and vascularized chamber model}

The vascularized groin chamber model consists of a silicone tube sleeved around the epigastric vessels that branch from the femoral artery and vein in the groin. ${ }^{12}$ After application of depilatory cream and decontamination of the skin with chlorhexidine and alcohol, the surgical field was exposed via a transverse incision above the inguinal fat pad. The superficial epigastric vessels were freed from surrounding tissue along a $1 \mathrm{~cm}$ length from their origin at the femoral vessels to their entry into the inguinal fat pad. Sili- cone tube chambers (Dow-Corning Corp.) cut to lengths of $5 \mathrm{~mm}$, with an internal diameter of $3.55 \mathrm{~mm}$ and a volume of $42 \mu \mathrm{L}$, were slit open on one side and placed around the epigastric vessels. A 10/0 Nylon microsuture stitch was placed at the proximal end of the chamber, anchoring it to the underlying muscle. The proximal end and side were then sealed with melted bone wax (Ethilon), leaving sufficient opening for the vessels to pass. After filling the chamber with its contents the distal end was also sealed in the same fashion. Finally, the wounds were closed with metal clips.

\section{LPC culture}

LPCs derived from an adult TAT GRE LacZ transgenic mouse were used in these studies. ${ }^{15}$ They were cultured at $37^{\circ} \mathrm{C}$ in $5 \% \mathrm{CO}_{2}$ in either Williams' E solution (Gibco, Cat. No. 12551032) containing antibiotics, $10 \%$ fetal calf serum, and epidermal growth factor $(50 \mathrm{ng} / \mathrm{mL})$ (Chemicon Int., Cat. No. 445042) and insulin $(10 \mu \mathrm{L} / \mathrm{mL})$, termed "growth medium," or the so-called "differentiation medium," which was further supplemented with Insulin-Transferrin-Selenium $10 \mu \mathrm{L} / \mathrm{mL}$ (Gibco, Cat. No. 41400045), nicotinamide $(1 \mu \mathrm{L} /$ $\mathrm{mL})$, and dexamethasone $(4 \mu \mathrm{L} / \mathrm{mL})$. Immediately before surgery, the LPCs were trypsinized, washed three times with phosphate-buffered saline, and centrifuged to remove the fetal calf serum. The cells were then aliquoted into units of $1 \times 10^{6}$ cells. After incubation for $5 \mathrm{~min}$ at $37^{\circ} \mathrm{C}$, the suspension was placed on ice for a further $10 \mathrm{~min}$. The cells were then washed three times in phosphate-buffered saline and the pellet resuspended in $50 \mu \mathrm{L}$ aliquots of Matrigel (BD Biosciences, Cat. No. 356234) kept at $4^{\circ} \mathrm{C}$ for seeding into the animal chambers.

The spheroids were prepared by culturing LPCs in Matrigel for 1 week before implantation. To achieve this, the cells were washed and counted aliquots of 50,000 LPCs suspended in $50 \mu \mathrm{L}$ Matrigel were placed in 96-well plates and maintained for a week at $37^{\circ} \mathrm{C}$ in $5 \% \mathrm{CO}_{2}$ in Williams' $\mathrm{E}$ solution, with and without the differentiation supplements described above. Spheroid formation was seen under low magnification light microscopy after 1 week (Fig. 1).

\section{Animal groups}

The animals were divided into four groups, each consisting of four mice. Animals in groups 3 and 4 were fed the CDE diet as described earlier, whereas those in groups 1 and 2 were kept on a normal diet. Chambers were implanted in the groin bilaterally in each animal and either contained $1 \times 10^{6}$ cells directly suspended in Matrigel (group 1, group 3) or spheroid formations that had formed from aliquots of 50,000 cells in culture in Matrigel over a period of 1 week (groups 2 and 4). The chamber in the right groin contained cells cultured with the supplement media as described above, whereas those on the left side were maintained in the growth medium (Fig. 2).

\section{Chamber harvest}

At 4 weeks after chamber implantation, mice were anesthetized using chloral hydrate given intraperitoneally at $4 \mathrm{mg} / \mathrm{g}$ body weight. A small incision was made over the original wound and the chamber dissected from its surrounding tissue. Patency of the pedicle was assessed quali- 

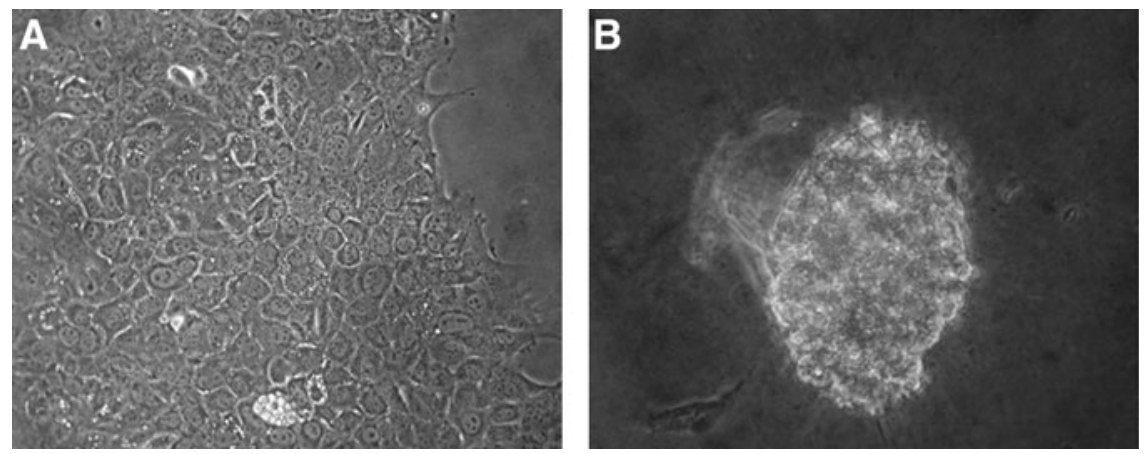

FIG. 1. Light microscopy images of LPCs in culture before implantation. (A) LPCs cultured in the growth medium forming a monolayer. (B) Spheroids formed in Matrigel after culture of LPCs for 7 days. Optical magnification $(\mathbf{A}): \times 10,(\mathbf{B}): \times 20$. LPCs, liver progenitor cells.

tatively before explantation of the construct. The mice were subsequently sacrificed with an overdose of Lethabarb (Virbec, Cat. No. 1P0643-1) given intraperitoneally. After removal of the silicone tubing, chamber tissues were weighed before processing.

\section{Histology and immunohistochemistry}

All specimens were fixed whole in $4 \%$ paraformaldehyde overnight. The fixed tissue was then processed to paraffin, and sections were cut longitudinally at $5 \mu \mathrm{m}$, placed on silanized slides, and dried overnight in a $37^{\circ} \mathrm{C}$ incubator. Histological staining was performed using hematoxylin and eosin (H\&E) to analyze general morphology and periodic acid Schiff (PAS) reaction to demonstrate glycogen storage in cells, and a total of six to seven chambers were sectioned and analyzed for each of the four groups.

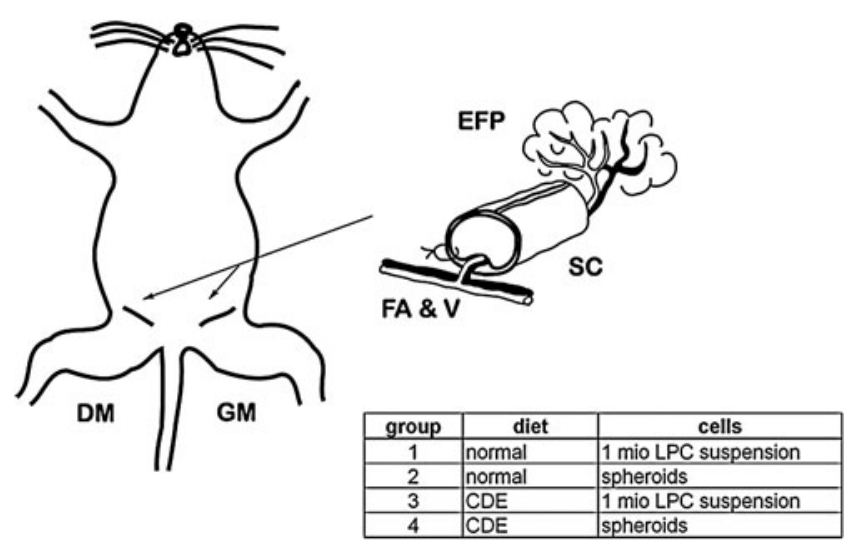

FIG. 2. Schematic illustration of experimental design and treatment groups. The animals were divided into four groups each consisting of four animals. Mice in groups 1 and 2 were fed a normal diet, whereas groups 3 and 4 received a $\mathrm{CDE}$ diet to induce liver injury. Bilateral silicone chambers contained either $1 \times 10^{6}$ LPCs directly suspended in Matrigel (groups 1 and 3), or spheroids cultured over 1 week from 50,000 LPCs (groups 2 and 4), which were placed around the epigastric vessels and sealed with bone wax. Cells in the constructs implanted in the right groin had been prepared in standard growth media, and those on the right differentiation media with supplemented nicotinamide and dexamethasone. CDE, choline-deficient ethionin-supplemented; DM, differentiation medium; EFP, epigastric fat pad; FA \& V, femoral artery and vein; GM, growth medium; SC, silicone chamber.
Immunohistochemical staining for Ki67 (Labvision rabbit monoclonal Ab, SP6 No. RM-9106) to assess proliferation of implanted LPCs was performed using a Dako Autostainer, on sections adjacent to those for H\&E and PAS where possible. After dewaxing and hydration, antigen retrieval was performed by heating sections in tris/EDTA buffer, $\mathrm{pH} 9.0$ at $95^{\circ} \mathrm{C}$ for $20 \mathrm{~min}$, followed by $20 \mathrm{~min}$ cool-down at room temperature. Slides were then loaded in to the machine for all subsequent steps. Endogenous peroxidise was first blocked with $3 \%$ peroxide in 50\% methanol for $5 \mathrm{~min}$, followed by protein blocking with 10\% normal goat serum for $30 \mathrm{~min}$. Primary antibody was applied at 1: 500 for $1 \mathrm{~h}$, followed by Vector biotinylated goat anti rabbit at 1:200 for $30 \mathrm{~min}$, and Dako HRP-streptavidin Enzyme activity was then observed with Thermo Ultravision DAB Plus substrate for $5 \mathrm{~min}$, and slides removed from the machine, counterstained in hematoxylin, dehydrated, cleared, and mounted in DePex. Tris buffered saline (TBS) was used as diluent for all reagents and wash steps, and all reactions took place at room temperature. Diluent alone was used in place of primary antibody as a reagent negative control.

All sections were viewed under light microscopy using Zeiss Axioskop 2 and Plan-NEOFLUAR lenses (1.25-100-fold magnification). Images were captured with a Zeiss AxioCam Mrc5.

\section{Statistical analysis}

Data were presented as mean with standard error for the mean. Statistical analysis was performed using two-way analysis of variance and differences with a $p$-value $>0.05$ were considered significant.

\section{Results}

\section{General observations}

All animals survived the duration of the study and those placed on the CDE diet tolerated it well. There were no perioperative complications. The vascular pedicles of all but one chamber, where it had thrombosed (group 3), were macroscopically patent and filled with vital tissue at the time of chamber harvest. The overall mean weight of the chamber contents varied only slightly within and also between the groups (Table 1). The highest mean tissue weight of $16.1 \mathrm{mg}$ (range 15.0-20.4 mg) was found in group 4 with animals on the CDE diet and implanted spheroids cultivated with the growth medium, whereas constructs grown from spheroids without supplement media in animals on a normal diet 
Table 1. Weights of Tissue Constructs Harvested from the Tissue Engineering Chamber AfTER 4 WEEKS

\begin{tabular}{|c|c|c|c|c|c|c|c|c|}
\hline \multirow[b]{3}{*}{ Animal } & \multicolumn{8}{|c|}{ Weight (mg) } \\
\hline & \multicolumn{2}{|c|}{ Group 1} & \multicolumn{2}{|c|}{ Group 2} & \multicolumn{2}{|c|}{ Group 3} & \multicolumn{2}{|c|}{ Group 4} \\
\hline & $G M$ & $D M$ & $G M$ & $D M$ & GM & $D M$ & $G M$ & $D M$ \\
\hline 1 & 18.3 & 10.7 & 10.4 & 8.4 & 12.9 & 20 & 15 & 11.6 \\
\hline 2 & 14.8 & 16.3 & 10.5 & 15 & 14.5 & 12.8 & 15 & 19.8 \\
\hline 3 & 16.2 & 19.4 & 14.2 & 5.3 & 6.5 & 11.7 & 20.4 & 19.8 \\
\hline 4 & 10 & 14.1 & 14.8 & 13.8 & $\mathrm{t}$ & 13 & 14 & 7.5 \\
\hline Mean & 14.83 & 15.13 & 12.47 & 10.63 & 11.3 & 14.38 & 16.1 & 14.68 \\
\hline SEM & 1.365 & 1.419 & 0.9107 & 1.768 & 1.728 & 1.469 & 1.125 & 2.382 \\
\hline
\end{tabular}

The overall mean weight of the chamber contents varied only slightly within and also between the groups. Animals in groups 3 and 4 were fed the CDE diet; animals in groups 1 and 2 were kept on a normal diet. Chambers were implanted in the groin bilaterally in each animal and either contained $1 \times 10^{6}$ cells in Matrigel (groups 1 and 3) or spheroid formations after culture of 50,000 cells for 1 week in Matrigel (groups 2 and 3). The chamber in the right groin contained cells previously cultured in the differentiation medium; those on the left side, maintained in the growth medium. The highest mean tissue weight was found in animals on the CDE diet with implanted spheroids cultivated in the differentiation medium. CDE, choline-deficient ethionin-supplemented; DM, differentiation medium; GM, growth medium; SEM, standard error for the mean; $t$, thrombosed.

(group 2) produced the lowest average weight of $10.62 \mathrm{mg}$ (range $8.4-13.8 \mathrm{mg}$ ). Neither diet nor cell culture technique had a statistically significant influence on the construct weight ( $p>0.05$ from two-way analysis of variance).

\section{Histomorphology}

The implanted LPCs were readily identified in H\&E sections based on their characteristic appearance, which included small size, ovoid, and intensely basophilic nuclei, and scant, though eosinophilic, cytoplasm (Fig. 3). The main components of all of the harvested tissue constructs aside from the identified LPCs were Matrigel remnants, adipocytes, vascular structures, both the original epigastric vascular pedicle-seen running longitudinally along one side of the construct (Fig. 4) and new vessels created via angiogenesis from the pedicle (Fig. 3c), and inflammatory cells in varying numbers.

The number of LPCs within the harvested constructs showed considerable variation between groups. Across all groups, under low power magnification it was not possible to detect a clear difference in cell number, morphology, distribution, or proliferation (Ki67 labeling) in chambers where implanted cells were cultured in growth compared to differentiation medium. The description that follows therefore considers these two subgroups together, for each of the four main groups.

Chamber tissue morphology in the two groups receiving dissociated cells (group 1, normal diet; group 3, CDE diet) was similar. The greatest number of LPCs was noted adjacent to the vascular pedicle (Fig. $4 \mathrm{a}, \mathrm{b}, \mathrm{e}, \mathrm{f}$ ), and most were
FIG. 3. (a) LPCs forming a line of cells (indicated by brackets) on either side of a capillary in longitudinal section (arrow). (b) LPCs in no particular arrangement. Both (a) and (b) are from group 3 (LPCs implanted as dissociated cells on CDE diet) and demonstrate the LPCs in high power as generally oval cells also note some heterogeneity in size and shape, and with a dense nucleus and a relatively small amount of dark pink cytoplasm. (c) Medium power view of the pedicle $(\mathrm{P})$ sprouting new capillaries (arrows) into the construct Matrigel surrounded by large clusters of LPCs. Further from the pedicle the Matrigel areas (M) are less cellular (from group 4, spheroidimplanted CDE diet chamber). (a-c) Hematoxylin and eosin-stained sections. Panels (a) and (b) were taken at $\times 100$ objective and scale bars $=20 \mu \mathrm{m}$; (c) was taken with $\times 10$ objective and the scale bar $=100 \mu \mathrm{m}$. Color images available online at www.liebertonline.com/ten.
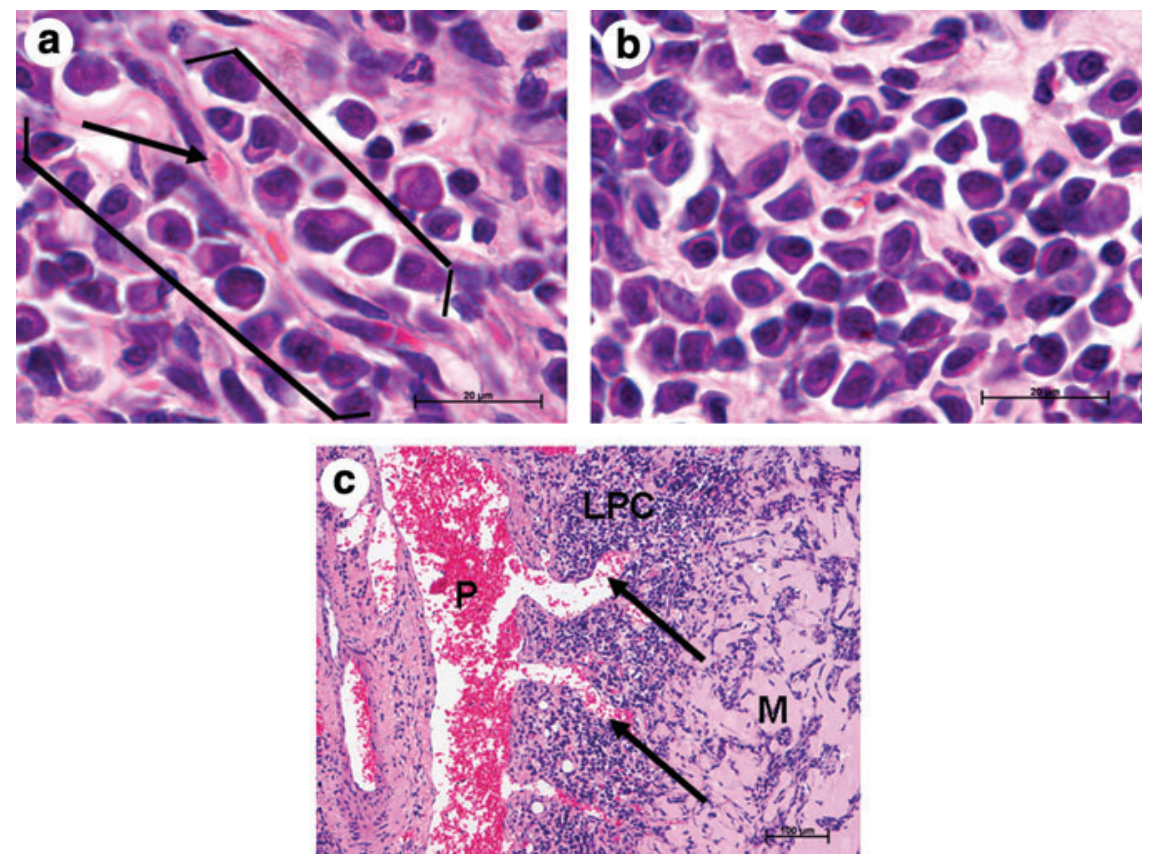


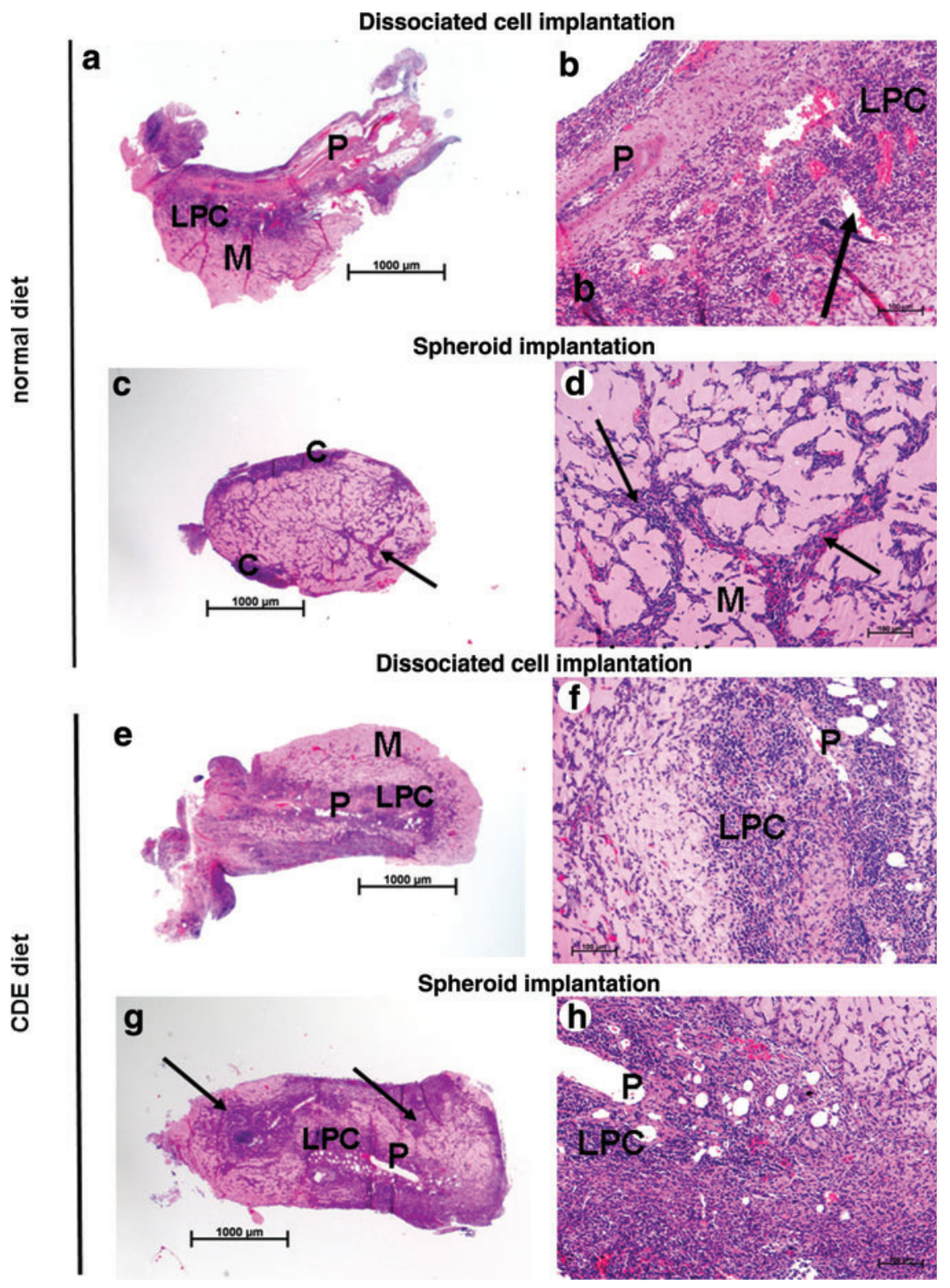

FIG. 4. Low-power and high-power micrographs of longitudinal sections showing characteristic construct appearance in all four groups: $(\mathbf{a}, \mathbf{b})$ dissociated cells, control diet; $(\mathbf{b}, \mathbf{d})$ spheroids in control diet; $(\mathbf{e}, \mathbf{f})$ dissociated cells with CDE diet; and $(\mathbf{g}, \mathbf{h})$ spheroids with CDE diet. The pedicle $(\mathrm{P})$ is seen running along the length of the construct in $(\mathbf{a}, \mathbf{e}, \mathbf{g})$. The pedicle is not seen in (c), which is a section some distance from the pedicle, although some capsule development $(\mathrm{C})$ is evident peripherally. In (a, $\mathbf{b}, \mathbf{e}-\mathbf{h})$, LPCs can be seen around the pedicle. Additionally in the spheroid groups (c, d), thick tracks of LPCs (arrows) are seen, and even larger groupings of LPCs ( $g$, arrows) can be seen at long distances from the pedicle. In the dissociated cell implantations (a, e) in low power it is event that the Matrigel (M) areas away from the pedicle are relatively acellular, whereas the spheroid-implanted chambers (c, g) are more cellular, including large masses of LPCs at large distances from the pedicle, particularly (g) which is also on the CDE diet. Arrow in (b) indicates capillary branch from the pedicle. Hematoxylin and eosin staining: (a, c, e, g), taken on $1.25 \times$ objective and scale bars $=1000 \mu \mathrm{m} ;(\mathbf{b}, \mathbf{d}, \mathbf{f}, \mathbf{h})$, taken on $10 \times$ objective and scale bars $100 \mu \mathrm{m}$. Color images available online at www.liebertonline.com/ten.

present as masses of varying size and density, and infiltrated with new capillaries growing from the pedicle (Fig. 4b). A few scattered, isolated cells were also noted in areas more distant from the pedicle. Varying numbers of inflammatory cells, consisting of lymphocytes and neutrophils, were noted in these chambers. These were usually located either scattered among the LPCs or more peripherally, where they formed part of the capsule lining the internal surface of the silicone chamber (Fig. 4c), most likely as a response to this rather than the implanted cells.

The LPCs seen in group 2 (normal diet, spheroids) were of a similar number to those in the two groups described above, despite fewer total cells implanted. In contrast, however, in several chambers the cells were arranged in tracts throughout the chamber, including areas a long distance from the pedicle. These LPC tracts included a central blood vessel and also contained other (i.e., non-LPC) cell types such as inflammatory cells. The tracts were separated by pockets of acellular Matrigel (Fig. 4c, d).
The chambers implanted in CDE-fed mice with spheroids (group 4) gave rise to larger and often denser LPC masses, with smaller amounts of residual Matrigel. This is noteworthy given that far fewer cells were implanted in the spheroid compared to single cell groups. As above, the cell masses tended to be focused around the pedicle (Fig. $4 \mathrm{~g}, \mathrm{~h}$ ), but also occurred elsewhere at considerable distances from the pedicle. The cell tracts noted in group 2 (spheroid implantation no diet) were not as evident here, and it may be that with greater survival and/or proliferation in group 4, larger masses rather than narrow tracts are formed. Inflammatory cells were as described previously.

In chambers from all groups, under high power magnification, cord-like arrangements of LPCs could be seen, somewhat reminiscent of the hepatic plates seen in mature liver (Fig. 3a). These were often arranged parallel to capillaries. In most instances, however, LPCs were loosely clustered and showed no obvious arrangement or close association (Fig. 3b), and this was the same in both single-cell and spheroid groups. 
Ki67 x10
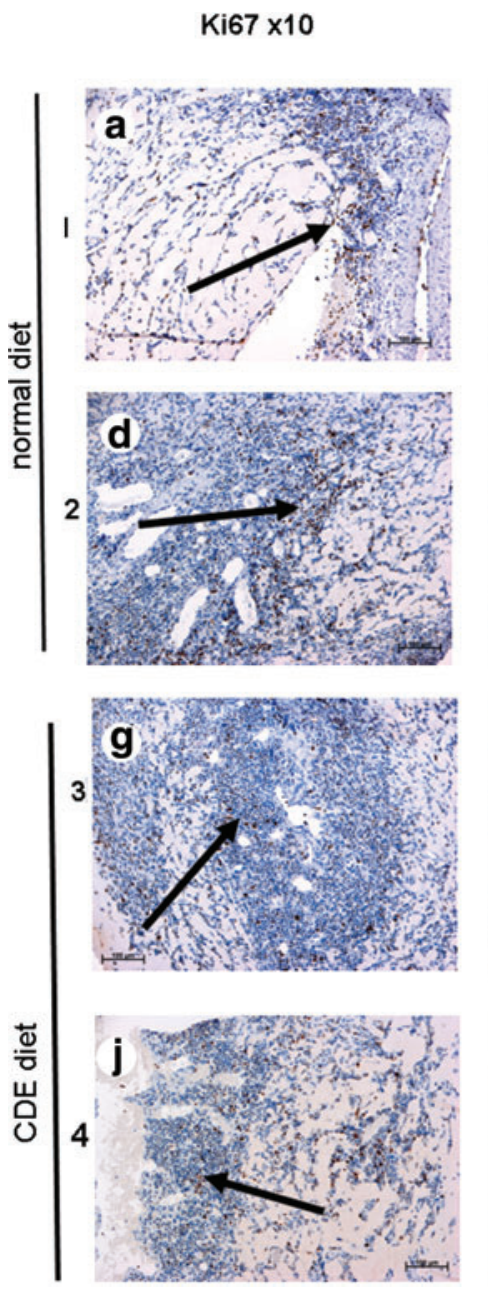

Ki67 $\times 40$

Dissociated cell implantation

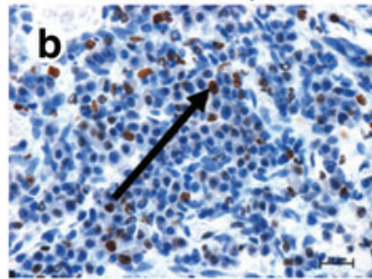

Spheroid implantation

micrographs from all four groups demonstrating

large numbers of proliferating Ki67-positive LPCs (arrows) in all four groups. (a, d, g, j), taken using $\times 10$ objective and scale bars = $100 \mu \mathrm{m} ;(\mathbf{b}, \mathbf{e}, \mathbf{h}$, k), taken using $\times 40$ objective and scale bars $=20 \mu \mathrm{m}$.

(c, f, i, 1) are high-power micrographs illustrating the magenta cytoplasm of

PAS-positive cells in each of the four groups; taken using a $\times 100$ objective under oil immersion, scale bars $=10 \mu \mathrm{m}$. PAS, periodic acid Schiff. Color images available online at www.liebertonline.com/ ten.

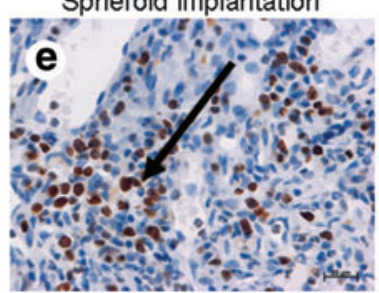

Dissociated cell implantation

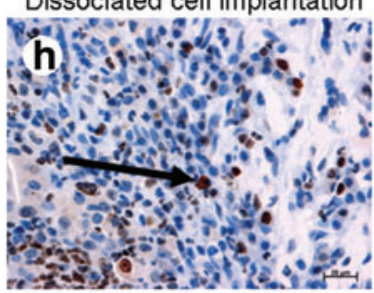

Spheroid implantation

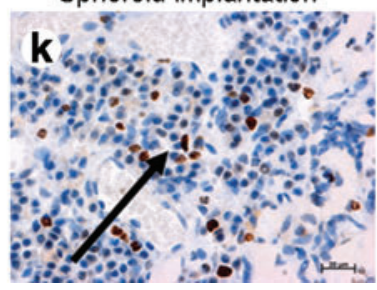

PAS $\times 100$
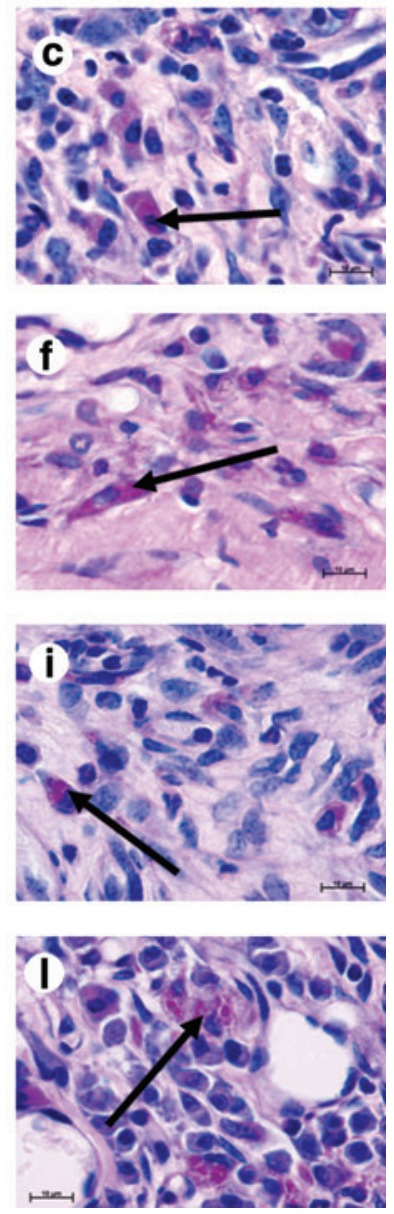

Some LPCs or LPC-derived cells were clearly enlarged and more cuboidal compared to LPCs with the standard morphology described earlier (Fig. 3a), presumably reflecting some degree of maturation toward a mature hepatocyte phenotype. No obvious ductal structures were seen.

\section{Proliferation and differentiation}

Ki67 immunostaining demonstrated, that in all four groups, a considerable number of the identified LPCs were clearly proliferating (Fig. 5a, b, d, e, g, h, j, k). As a percentage of LPCs (identified by morphology) the percentage of proliferating cells are similar across the different experimental groups ranging between $10 \%$ and $15 \%$. A far higher number of proliferating cells were observed among LPC cells than in other regions of the chamber construct that did not include LPCs.

As a measure of possible differentiation of the implanted LPCs toward a mature hepatocyte phenotype, samples were stained with PAS to detect glycogen production. In each of the four groups, it was estimated that $\sim 5 \%-10 \%$ of identified LPCs contained PAS-positive material (Fig. 5c, f, i, l). The PAS-positive cells as a percentage of total LPCs appeared to be similar for the four groups. Some of the positive cells were larger due to an expanded glycogen-containing cytoplasm, and possessed the more mature appearance described above. Staining in a given cell was sometimes granular and other times more homogeneous, but was always distinctly a dark magenta compared to the pale pink of the extracellular matrix and Matrigel-containing background (Fig. 5c, f, i, 1).

\section{Discussion}

The treatment of end-stage liver failure continues to pose a therapeutic challenge. Despite the success of liver transplantation, an increasing lack of donor organs has directed much research toward the use of cell-based therapies, including direct transplantation of mature hepatocytes into the spleen or portal vasculature ${ }^{4}$ and extra- or intracorporeal bioartificial liver devices. ${ }^{7}$ Direct transplantation of hepatocytes via the portal venous system is one clinically established method. Unfortunately, embolization of cells into the spleen or liver itself is associated with portal hypertension and transient ischemia reperfusion injury. Further, up to $70 \%$ of the transplanted cells remain intravascularly trapped and undergo phagocytosis with a subsequent significant loss of cell count. ${ }^{16}$

Ideally, a liver replacement system would consist of a construct containing a mass of cells with a high, but controllable proliferation and differentiation capacity, which is able to compensate for the organ's diminished functional properties indefinitely. Its application should also cause 
minimal morbidity and not rely on additional treatments such as immunosuppressants.

Thus far, all of the experimental cell-based treatment modalities for liver failure share the same problem, namely, the difficulty of culturing and expanding hepatocytes to achieve sufficient numbers for functional organ replacement. To overcome this obstacle, research is moving toward the use of LPCs. Unlike hepatocytes, which tend to lose their proliferative capacity, dedifferentiate and even undergo apoptosis when their intercellular attachments are disrupted during isolation for culture in vitro, LPCs are much more easily isolated, cultured, and expanded. ${ }^{17,18}$

The tissue engineering chamber used in our study is an established model for the in vivo generation of tissue constructs on a defined vascular pedicle from cells of different lineages. Studies conducted at our institute have successfully shown adipogenesis, ${ }^{11,19}$ survival, and differentiation of both pituitary colony-forming cells, ${ }^{20}$ survival and function of pancreatic islets, ${ }^{21}$ and survival of adipose-derived mesenchymal stem cells, ${ }^{22}$ and the formation of myotubes from myoblasts (unpublished data) using the chamber construct. In the context of cell-based liver replacement, this localized and controllable environment could therefore potentially be an ideal vehicle to avoid the problems encountered with intravascular cell applications.

To replicate the situation of liver failure, the mice in two of the four groups used in this study were put on a CDE diet for 1 month before treatment. This is a well-established model for chronic liver injury and it is also known to induce proliferation and differentiation of LPCs within the damaged organ. ${ }^{14,15}$ We hypothesized that as well as the intrahepatic signals activating LPCs, there might also be a systemic component that could affect the cells within the chamber. The finding that the chambers implanted with spheroids in CDEtreated mice produced constructs with the greatest number of LPC and hepatocyte-like cells supports this notion.

As organs are three-dimensional structures, the survival and function of the cellular components rely on maintained physiological surroundings. Especially mature hepatocytes are known to be greatly dependent on intercellular attachment and signaling for survival. ${ }^{5,6,23}$ It is therefore not surprising that the LPCs as hepatocyte precursors also thrive when cultivated in a three-dimensional matrix to resemble the microanatomy of normal liver tissue more closely. In this study the implantation of freshly suspended LPCs in Matrigel directly into the chamber clearly showed cell proliferation and differentiation, but the extent of these events was clearly increased when the cells were first cultured to form threedimensional clusters within a matrix (in this case Matrigel) before implantation. Despite the fact that the implanted spheroids were cultured starting out with units of only 50,000 LPCs, versus $1 \times 10^{6}$ LPCs used for direct implantation, more LPCs cells were found in the spheroid chambers at 4 weeks. This would imply that in view of a potential clinical application, the amount of initial donor cells needed could be significantly reduced by induction of spheroid formation before transplantation. Supplementation of the culture media with dexamethasone and nicotinamide (termed differentiation medium) seemed to have no obvious effect on cell survival or differentiation of the LPCs to more mature cell forms.

In some areas of the chamber, the ovoid cells aligned in a distinct single-file pattern, reminiscent of how hepatocytes follow the hepatic sinusoids. The moderate inflammatory response within the tissue constructs and the strong induction of vascularization are also indications that this chamber model can provide a sustainable environment, which facilitates three-dimensional association of the cells to form a functional organoid.

LPCs are at least bipotential as they can differentiate into both cholangiocytes and hepatocytes. The specific signals that determine the alternate paths of differentiation are yet to be defined. Under the conditions used in our study, $5 \%-10 \%$ of LPCs demonstrated signs of functional differentiation to hepatocytes, based on cytoplasmic glycogen storage (PAS). Many of these PAS-positive cells were larger and appeared more mature (larger of more cuboidal shape) than the ovoid LPCs. None of the tissue sections obtained in this study showed obvious ductal formations. Collectively, the data suggest that the environment provided to the chamber in a CDE-treated mouse favors the growth and differentiation of LPCs toward the hepatocyte lineage, as well as the maintenance of some LPCs. It is possible that the milieu that exists in damaged and inflamed liver contributes toward a favorable environment, which allows for differentiation of LPCs to both hepatocyte and cholangiocytes. ${ }^{24}$ This is also supported by the finding that bone marrow cells can differentiate into hepatocytes when exposed to extracts from damaged hepatocytes. ${ }^{25}$

In this study we show that LPCs can survive, proliferate, and differentiate into hepatocytic cells in vivo. By combining the tissue engineering chamber model, with the threedimensional culture of LPCs into spheroids in a matrix, we provide a novel delivery method for introducing cells. In this environment, which favors their proliferation and differentiation toward mature hepatocytes, a good yield of cells with a mature hepatocytic phenotype can be achieved. For this cell-based liver replacement system to be useful as a viable alternative to whole-organ transplantation, the issue of incorporating or inducing the formation of a ductal drainage system would have to be addressed. However, it is feasible that this chamber system could be applied in genetic or metabolic diseases where pure hepatocyte-specific synthetic functions are required, such as clotting factors or enzymes. As a next step, we aim to consolidate these encouraging findings through further studies including longer time points, in depth functional arrays of the hepatocytic cells, and also investigations into alternative matrices to Matrigel that could be applied in humans.

\section{Acknowledgments}

We wish to thank Sue McKay, Liliana Pepe, and Anna Deftereos for their surgical assistance.

\section{Disclosure Statement}

No competing financial interests exist.

\section{References}

1. Taub, R. Liver regeneration: from myth to mechanism. Nat Rev Mol Cell Biol 5, 836, 2004.

2. Groth, C.G., Arborgh, B., Bjorken, C., Sundberg, B., and Lundgren, G. Correction of hyperbilirubinemia in the glucuronyltransferase-deficient rat by intraportal hepatocyte transplantation. Transplant Proc 9, 313, 1977. 
3. Fausto, N. Liver regeneration and repair: hepatocytes, progenitor cells, and stem cells. Hepatology 39, 1477, 2004.

4. Weber, A., Groyer-Picard, M.T., Franco, D., and Dagher, I. Hepatocyte transplantation in animal models. Liver Transpl 15, 7, 2009.

5. Mesnil, M., Fraslin, J.M., Piccoli, C., Yamasaki, H., and Guguen-Guillouzo, C. Cell contact but not junctional communication (dye coupling) with biliary epithelial cells is required for hepatocytes to maintain differentiated functions. Exp Cell Res 173, 524, 1987.

6. Rivera, D.J., Gores, G.J., Misra, S.P., Hardin, J.A., and Nyberg, S.L. Apoptosis by gel-entrapped hepatocytes in a bioartificial liver. Transplant Proc 31, 671, 1999.

7. Fiegel, H.C., Kaufmann, P.M., Bruns, H., Kluth, D., Horch, R.E., Vacanti, J.P., and Kneser, U. Hepatic tissue engineering: from transplantation to customized cell-based liver directed therapies from the laboratory. J Cell Mol Med 12, 56, 2008.

8. Paku, S., Schnur, J., Nagy, P., and Thorgeirsson, S.S. Origin and structural evolution of the early proliferating oval cells in rat liver. Am J Pathol 158, 1313, 2001.

9. Fougere-Deschatrette, C., Imaizumi-Scherrer, T., StrickMarchand, H., Morosan, S., Charneau, P., Kremsdorf, D., Faust, D.M., and Weiss, M.C. Plasticity of hepatic cell differentiation: bipotential adult mouse liver clonal cell lines competent to differentiate in vitro and in vivo. Stem Cells 24, 2098, 2006.

10. Ogawa, K., Ochoa, E.R., Borenstein, J., Tanaka, K., and Vacanti, J.P. The generation of functionally differentiated, three-dimensional hepatic tissue from two-dimensional sheets of progenitor small hepatocytes and nonparenchymal cells. Transplantation 77, 1783, 2004.

11. Lee, H., Cusick, R.A., Utsunomiya, H., Ma, P.X., Langer, R., and Vacanti, J.P. Effect of implantation site on hepatocytes heterotopically transplanted on biodegradable polymer scaffolds. Tissue Eng 9, 1227, 2003.

12. Cronin, K.J., Messina, A., Knight, K.R., Cooper-White, J.J., Stevens, G.W., Penington, A.J., and Morrison, W.A. New murine model of spontaneous autologous tissue engineering, combining an arteriovenous pedicle with matrix materials. Plast Reconstr Surg 113, 260, 2004.

13. Akhurst, B., Croager, E.J., Farley-Roche, C.A., Ong, J.K., Dumble, M.L., Knight, B., and Yeoh, G.C. A modified cholinedeficient, ethionine-supplemented diet protocol effectively induces oval cells in mouse liver. Hepatology 34, 519, 2001.

14. Viebahn, C.S., and Yeoh, G.C. What fires prometheus? The link between inflammation and regeneration following chronic liver injury. Int J Biochem Cell Biol 40, 855, 2008.

15. Tirnitz-Parker, J.E., Tonkin, J.N., Knight, B., Olynyk, J.K., and Yeoh, G.C. Isolation, culture and immortalisation of hepatic oval cells from adult mice fed a choline-deficient, ethioninesupplemented diet. Int J Biochem Cell Biol 39, 2226, 2007.
16. Joseph, B., Malhi, H., Bhargava, K.K., Palestro, C.J., McCuskey, R.S., and Gupta, S. Kupffer cells participate in early clearance of syngeneic hepatocytes transplanted in the rat liver. Gastroenterology 123, 1677, 2002.

17. Lemire, J.M., and Fausto, N. Multiple alpha-fetoprotein RNAs in adult rat liver: cell type-specific expression and differential regulation. Cancer Res 51, 4656, 1991.

18. Kakinuma, S., Nakauchi, H., and Watanabe, M. Hepatic stem/progenitor cells and stem-cell transplantation for the treatment of liver disease. J Gastroenterol 44, 167, 2009.

19. Kelly, J.L., Findlay, M.W., Knight, K.R., Penington, A., Thompson, E.W., Messina, A., and Morrison, W.A. Contact with existing adipose tissue is inductive for adipogenesis in Matrigel. Tissue Eng 12, 2041, 2006.

20. Lepore, D.A., Thomas, G.P., Knight, K.R., Hussey, A.J., Callahan, T., Wagner, J., Morrison, W.A., and Thomas, P.Q. Survival and differentiation of pituitary colony-forming cells in vivo. Stem Cells 25, 1730, 2007.

21. Hussey, A.J., Winardi, M., Han, X.L., Thomas, G.P., Penington, A.J., Morrison, W.A., Knight, K.R., and Feeney, S.J. Seeding of pancreatic islets into prevascularized tissue engineering chambers. Tissue Eng Part A 15, 3823, 2009.

22. Choi, Y.S., Matsuda, K., Dusting, G.J., Morrison, W.A., and Dilley, R.J. Engineering cardiac tissue in vivo from human adipose-derived stem cells. Biomaterials 31, 2236, 2010.

23. Bhatia, S.N., Balis, U.J., Yarmush, M.L., and Toner, M. Probing heterotypic cell interactions: hepatocyte function in microfabricated co-cultures. J Biomater Sci Polym Ed 9, 1137, 1998.

24. Viebahn, C.S., Benseler, V., Holz, L.E., Elsegood, C.L., Vo, M., Bertolino, P., Ganss, R., and Yeoh, G.C. Invading macrophages play a major role in the liver progenitor cell response to chronic liver injury. J Hepatol 53, 500, 2010.

25. Kuo, T.K., Hung, S.P., Chuang, C.H., Chen, C.T., Shih, Y.R., Fang, S.C., Yang, V.W., and Lee, O.K. Stem cell therapy for liver disease: parameters governing the success of using bone marrow mesenchymal stem cells. Gastroenterology 134, 2111, 2008.

Address correspondence to: Wayne A. Morrison, M.D., B.S. Bernard O'Brien Institute of Microsurgery St. Vincent's Hospital 42 Fitzroy St. Fitzroy 3065 Victoria Australia

E-mail: wayne.morrison@unimelb.edu.au

Received: July 26, 2009

Accepted: October 12, 2010

Online Publication Date: December 10, 2010 